\title{
Lançamento oblíquo com resistência do ar: Uma análise qualitativa
}

\author{
Oblique launch with air resistance: A qualitative analysis
}

\author{
Wilson Hugo C. Freire, Marciano L. Medeiros, Daniela Leite, Raffaela M. Silva \\ Departamento de Física, Universidade Regional do Cariri, Juazeiro do Norte, Ceará, Brasil
}

Recebido em 11 de agosto de 2015. Aceito em 30 de setembro de 2015

\begin{abstract}
Consideramos o movimento de uma partícula lançada obliquamente sob ação da gravidade e de uma força de resistência do ar proporcional à velocidade. Obtemos, via cálculo diferencial, informações de caráter geral sobre a trajetória da partícula como intervalos de crescimento (subida) e decrescimento (queda), ponto de máximo (altura máxima), concavidade, comportamento assintótico, alcance horizontal etc., o que nos permite traçar o perfil desta trajetória sem a utilização de tabelas numéricas e sem a necessidade de fazer aproximações. Apresentamos uma demonstração de que, apesar de não haver uma expressão exata explícita para o alcance horizontal da partícula, este alcance é sempre menor quanto maior for o valor do parâmetro de resistência do ar, o que condiz com a situação física em consideração.
\end{abstract}

Palavras-chave: lançamento oblíquo, resistência do ar, trajetória, alcance horizontal.

We consider the motion of a particle launched obliquely under gravity force and air resistance proportional to velocity. We obtain, via differential calculus, general informations about the trajectory of the particle as increasing and decrease intervals, maximum point, concavity, asymptotic behavior, horizontal reach etc., allowing us tracing the profile of this trajectory without the use of numerical tables and without the need to make approximations. We present a demonstration that, although there is no explicit exact expression for the horizontal reach of the particle, it is always smaller as larger the value of air resistance parameter, which matches the physical situation under consideration.

Keywords: oblique launch, air resistance, trajectory, horizontal reach.

\section{Introdução}

Uma situação física bastante comum no nosso dia a dia envolve o lançamento vertical ou oblíquo de objetos, por exemplo ao arremessarmos bolinhas de papel numa mesa ou num cesto. O lançamento oblíquo de uma partícula é um exemplo bastante ilustrativo de aplicação da mecânica newtoniana, envolve gravidade e atrito (no caso a resistência do ar) [1]- 3]. Além disso, o estudo do lançamento oblíquo tem importância em engenharia, por exemplo em situações onde se utilizam explosivos para desobstruir passagens, em lançamento de foguetes e também em balística.

Este trabalho é uma tentativa de apresentar uma proposta didática auxiliar ou complementar de abor-

*Endereço de correspondência: wilsonhugo1@gmail.com dagem do lançamento oblíquo com atrito do ar proporcional à velocidade. Embora abordado em vários livros-textos de mecânica clássica [1] - 3] e em outros trabalhos [4]- [5] nossa análise tem como foco informações gerais sobre este movimento e, para tal, utilizaremos conceitos e teoremas do cálculo diferencial. A partir da equação da trajetória, que advém da solução das equações de movimento da partícula, investigamos ponto de máximo, intervalos de crescimento e decrescimento, concavidade, assíntota etc. Isto nos permitirá esboçar a trajetória da partícula sem a necessidade de fazer tabelas numéricas ou aproximações.

Vale salientar que o uso de conceitos e teoremas de matemática em física pode trazer benefícios para ambas, além de um entendimento mais amplo de conceitos físicos. Como simples exemplo temos o 
fato de que o conhecimento da operação produto escalar nos permite determinar o trabalho de uma força constante, todavia o conhecimento do conceito de integral curvilínea nos permite determinar o trabalho no caso geral em que a força é variável. Neste artigo, veremos por exemplo que, mesmo sem haver uma expressão exata para o alcance horizontal da partícula, podemos mostrar, com o uso da derivada de funções implícitas, que o alcance é menor quanto maior for o atrito, o que é fisicamente esperado.

\section{Equações de movimento}

Vamos considerar o problema de uma partícula de massa $m$ lançada obliquamente com velocidade (inicial) de módulo $v_{0}>0$ segundo um ângulo $\theta \in(0 ; \pi / 2)$, em relação ao solo suposto inercial (Fig. 1).

Vamos considerar que a partícula, uma vez lançada, estará sob ação da força peso

$$
\mathbf{F}=-m g \hat{j} \quad(g=\text { constante }),
$$

e de uma força de atrito do ar, que vamos supor ser proporcional à velocidade

$$
\mathbf{f}=-b \mathbf{v}
$$

onde $b>0$ é constante.

A equação de movimento newtoniana desta partícula é dada por [1]- [3]

$$
\frac{d^{2} \mathbf{r}}{d t^{2}}=\frac{d \mathbf{v}}{d t}=\frac{1}{m}(\mathbf{F}+\mathbf{f})=-\beta\left(\frac{g}{\beta} \hat{j}+\mathbf{v}\right),
$$

onde $\beta=b / m$ que, pela relação com $b$ ( $m$ fixo), vamos nos referir como parâmetro de atrito do ar. As condições iniciais em questão são dadas por

$$
\mathbf{r}(0)=\mathbf{0}, \quad \mathbf{v}(0)=v_{0} \cos \theta \hat{i}+v_{0} \operatorname{sen} \theta \hat{j} .
$$

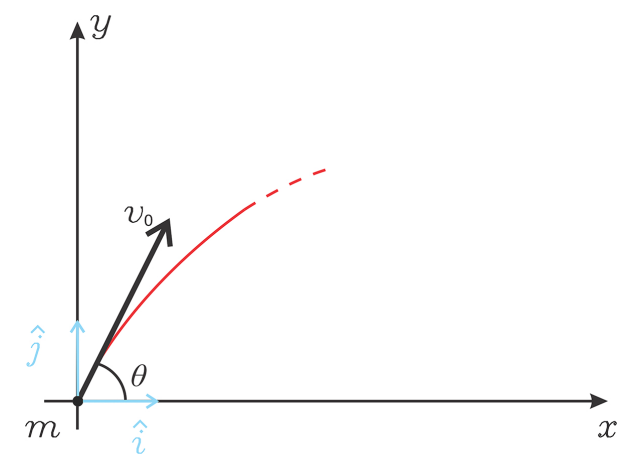

Figura 1: Lançamento da partícula.
Este problema pode ser reescrito em termos das componentes cartesianas do seguinte modo

$$
\begin{gathered}
\frac{d^{2} x}{d t^{2}}=\frac{d v_{x}}{d t}=-\beta v_{x}, \\
\frac{d^{2} y}{d t^{2}}=\frac{d v_{y}}{d t}=-\beta\left(v_{y}+\frac{g}{\beta}\right), \\
v_{x}(0)=v_{0} \cos \theta, \quad v_{x}(0)=v_{0} \operatorname{sen} \theta, \\
x(0)=y(0)=0 .
\end{gathered}
$$

As componentes da velocidade podem ser obtidas por uma primeira integração das Eqs. (5) e (6) com as condições iniciais (7) e são dadas por

$$
\begin{gathered}
v_{x}(t)=\left(v_{0} \cos \theta\right) e^{-\beta t}, \\
v_{y}(t)=\left(v_{0} \operatorname{sen} \theta+\frac{g}{\beta}\right) e^{-\beta t}-\frac{g}{\beta} .
\end{gathered}
$$

$\mathrm{Na}$ ausência de atrito do ar $(\beta \mapsto 0)$, a primeira destas duas últimas equações torna-se

$$
v_{x}(t)=v_{0} \cos \theta
$$

mas a Eq. 10 requer algum cuidado pois $\beta \neq 0$ se apresenta nos denominadores. No entanto, podemos proceder como segue

$$
\lim _{\beta \mapsto 0} v_{y}=v_{0} \operatorname{sen} \theta+g \lim _{\beta \mapsto 0} \frac{e^{-\beta t}-1}{\beta},
$$

e, usando a regra de l'Hospital (vide seção 5.4 da Ref. [6] ou seção 8.1 da Ref. [7]),

$$
\lim _{\beta \mapsto 0} v_{y}=v_{0} \operatorname{sen} \theta+g \lim _{\beta \mapsto 0} \frac{\left(-t e^{-\beta t}\right)}{1}=v_{0} \operatorname{sen} \theta-g t .
$$

Agora, integrando as Eqs. (9) e (10) com as condições iniciais (8) obtemos

$$
\begin{gathered}
x(t)=v_{0} \cos \theta\left(\frac{1-e^{-\beta t}}{\beta}\right), \\
y(t)=\left(v_{0} \operatorname{sen} \theta+\frac{g}{\beta}\right)\left(\frac{1-e^{-\beta t}}{\beta}\right)-\frac{g}{\beta} t .
\end{gathered}
$$

Aqui podemos obter o limite com $\beta \mapsto 0$ (correspondente a ausência de atrito do ar) mediante o uso da regra de l'Hospital, de forma semelhante a descrita anteriormente. Temos, para a componente $x$, 


$$
\begin{aligned}
\lim _{\beta \mapsto 0} x & =\left(v_{0} \cos \theta\right)\left(\lim _{\beta \mapsto 0} \frac{1-e^{-\beta t}}{\beta}\right)= \\
& =\left(v_{0} \cos \theta\right)\left(\lim _{\beta \mapsto 0} \frac{t e^{-\beta t}}{1}\right)=\left(v_{0} \cos \theta\right) t
\end{aligned}
$$

e, para a componente $y$,

$$
\begin{array}{r}
\lim _{\beta \mapsto 0} y=v_{0} \operatorname{sen} \theta\left(\lim _{\beta \mapsto 0} \frac{1-e^{-\beta t}}{\beta}\right)-g \\
\left(\lim _{\beta \mapsto 0} \frac{-1+e^{-\beta t}+t \beta}{\beta^{2}}\right)= \\
=\left(v_{o} \operatorname{sen} \theta\right) t-g\left(\lim _{\beta \mapsto 0} \frac{-t e^{-\beta t}+t}{2 \beta}\right)= \\
=\left(v_{o} \operatorname{sen} \theta\right) t-g\left(\lim _{\beta \mapsto 0} \frac{t^{2} e^{-\beta t}}{2}\right) \\
\therefore \lim _{\beta \mapsto 0} y=\left(v_{o} \operatorname{sen} \theta\right) t-\frac{g}{2} t^{2} .
\end{array}
$$

Note que as Eqs. (11), (12), 15) e (16), referentes ao caso sem resistência do ar, constam em livros de física geral, por ex. na Ref. [9].

\section{Trajetória}

Das Eqs. (13) e (14) temos que a equação da trajetória da partícula é dada por

$y(x)=\left(\tan \theta+\frac{g}{\beta v_{0} \cos \theta}\right) x+\frac{g}{\beta^{2}} \ln \left(1-\frac{\beta x}{v_{0} \cos \theta}\right)$.

Podemos esboçar o perfil desta trajetória sem fazer tabelas numéricas nem aproximações. Inicialmente notemos que, pela Eq. (9),

$$
\dot{x}(t)=v_{x}(t)=\left(v_{0} \cos \theta\right) e^{-\beta t}>0,
$$

logo $x(t)$ é estritamente crescente [6]- [7]; além disso

$$
x(0)=0 \quad \text { e } \lim _{t \mapsto+\infty} x(t)=\frac{v_{0} \cos \theta}{\beta} .
$$

Assim os valores da coordenada $x$ crescem no intervalo $0 \leqslant x<\xi \equiv\left(v_{0} \cos \theta\right) / \beta$. Notemos que a condição $x<\xi=\left(v_{0} \cos \theta\right) / \beta$ é essencial para que o logaritmo em $y(x)$ dada pela Eq. (17) faça sentido. Além do mais, tomando o limite de $y(x) \operatorname{com} x \mapsto \xi^{-}$ na Eq. (17) temos

$$
\lim _{x \mapsto \xi^{-}} y(x)=-\infty .
$$

Logo a trajetória da partícula tende a uma assíntota vertical dada por

$$
x=\xi=\frac{v_{0} \cos \theta}{\beta},
$$

caso não haja colisão da partícula com o solo no nível $y=0$. Notemos também que no caso sem atrito temos $0 \leqslant x<+\infty=\lim _{\beta \mapsto 0}\left[\left(v_{0} \cos \theta\right) / \beta\right]$ e não haveria tal assíntota.

\subsection{Intervalos de subida e de queda}

Derivando a equação da trajetória (Eq. (17)) com respeito a $x$ obtemos

$$
y^{\prime}(x)=\left(\tan \theta+\frac{g}{\beta v_{0} \cos \theta}\right)-\frac{g}{\beta} \cdot \frac{1}{v_{0} \cos \theta-\beta x} .
$$

Os intervalos de subida e de queda da partícula correspondem aos intervalos de crescimento e decrescimento da função $y(x)$, os quais correspondem respectivamente as condições $y^{\prime}(x)>0$ e $y^{\prime}(x)<0 \quad[6]$ [7]. Considerando a Eq. (20), a condição $y^{\prime}(x)>0$ implica em

$$
\frac{\beta v_{0} \operatorname{sen} \theta+g}{v_{0} \cos \theta}>\frac{g}{v_{0} \cos \theta-\beta x}
$$

e, como $v_{0} \cos \theta-\beta x>0$ (pois, pela Eq. (13), $\left.v_{0} \cos \theta-\beta x=v_{0} \cos \theta e^{-\beta x}>0\right)$, então

$$
\left(\beta v_{0} \operatorname{sen} \theta+g\right)\left(v_{0} \cos \theta-\beta x\right)>g v_{0} \cos \theta,
$$

ou seja,

$$
\beta x\left(\beta v_{0} \operatorname{sen} \theta+g\right)<\beta v_{0}^{2} \cos \theta \operatorname{sen} \theta
$$

de modo que

$$
x<\frac{v_{0}^{2} \operatorname{sen} \theta \cos \theta}{g+\beta v_{0} \operatorname{sen} \theta} .
$$

Assim a função $y(x)$ que descreve a trajetória da partícula é crescente (partícula subindo) para

$$
0<x<\frac{v_{0}^{2} \operatorname{sen} \theta \cos \theta}{g+\beta v_{0} \operatorname{sen} \theta} .
$$

De forma análoga obtemos que $y(x)$ é decrescente (partícula caindo) para

$$
\frac{v_{0}^{2} \operatorname{sen} \theta \cos \theta}{g+\beta v_{0} \operatorname{sen} \theta}<x<\xi=\frac{v_{0} \cos \theta}{\beta} .
$$


Também não é difícil obter que o ponto

$$
x_{c}=\frac{v_{0}^{2} \operatorname{sen} \theta \cos \theta}{g+\beta v_{0} \operatorname{sen} \theta}
$$

anula a derivada $y^{\prime}(x)$, ou seja, $y^{\prime}\left(x_{c}\right)=0$. Logo $x_{c}$ corresponde ao ponto crítico de máximo (global) da trajetória da partícula, visto que ela sobe até este ponto e depois segue caindo. A concavidade da trajetória pode ser determinada pela derivada segunda de $y(x)$. Da Eq. 20) obtemos

$$
y^{\prime \prime}(x)=-\frac{g}{\left(v_{0} \cos \theta-\beta x\right)^{2}}<0,
$$

que, além de reconfirmar a maximalidade do ponto crítico $x_{c}$, nos diz que a trajetória é côncava para baixo, conforme fisicamente esperado.

\subsection{Perfil da trajetória}

Podemos esboçar o perfil da trajetória $y(x)$ da partícula com base nas informações anteriormente obtidas; temos que $y(x)$

- é crescente para $0 \leqslant x<x_{c}$;

- tem um máximo para $x=x_{c}$;

- é decrescente para $x_{c}<x<\xi$;

- possui a assíntota vertical $x=\xi$;

- é sempre côncava para baixo.

A trajetória da partícula tem então o perfil esquematizado na Fig. 2.

\subsection{Alcance}

Neste contexto podemos mostrar que existe um único $A \in\left(x_{c} ; \xi\right)$, chamado alcance horizontal, tal que $y(A=0)$. De fato, temos que: $y(x)$ cresce no intervalo $0 \leqslant x<x_{c}$ de modo que $0 \leqslant y(x)<y\left(x_{c}\right)$

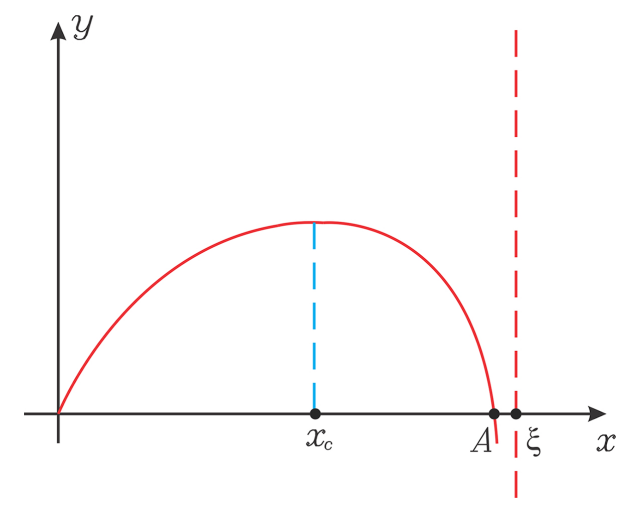

Figura 2: Perfil da trajetória. e então $y\left(x_{c}\right)>0$. Além disso $y(x)$ decresce para $x_{c}<x<\xi$ de modo que, tendo em vista a Eq. (18), há valores de $x$ neste intervalo tais que $y(x)<0$. Como $y(x)$ é contínua (e derivável) em $(0 ; \xi)$ então, pelo teorema do valor intermediário (vide seção 3.5 da Ref. [6] ou seção 7.2 da Ref. [7]), existe um $A \in\left(x_{c} ; \xi\right)$ tal que $y(A)=0$. A unicidade deste $A$ vem do fato de que toda função estritamente decrescente num intervalo aberto é injetiva neste intervalo. Assim a partícula atinge o solo (nível $y=0$ ) no ponto $x=A$ em $\left(x_{c} ; \xi\right)$. Entretanto não há uma expressão geral fechada para o alcance horizontal $A$. Ele deve satisfazer a seguinte condição (ver Eq. (17))

$$
\left(\tan \theta+\frac{g}{\beta v_{0} \cos \theta}\right) A+\frac{g}{\beta^{2}} \ln \left(1-\frac{\beta A}{v_{0} \cos \theta}\right)=0 .
$$

Mesmo assim, um fato físico que pode ser deduzido daqui, de forma geral, é que quanto maior o parâmetro de atrito $\beta$ menor será o alcance $A$. Ou seja, a função implícita $A=A(\beta)$ dada pela Eq. (21) é decrescente, isto é, satisfaz a condição

$$
\frac{d A}{d \beta}<0
$$

Para mostrar isto recorremos a regra de derivação de funções implícitas (vide seção 4.3 da Ref. [6]). Aplicando esta regra a Eq. (21), obtemos

$$
\begin{gathered}
\left\{\tan \theta+\frac{g}{\beta v_{0} \cos \theta}-\frac{g}{\beta\left(v_{0} \cos \theta-\beta A\right)}\right\} \frac{d A}{d \beta}= \\
=\frac{A \cdot g}{\beta^{2}}\left[\frac{1}{v_{0} \cos \theta}+\frac{1}{v_{0} \cos \theta-\beta A}\right]+ \\
\frac{2 g}{\beta^{3}} \ln \left(1-\frac{\beta A}{v_{0} \cos \theta}\right)
\end{gathered}
$$

Resta mostrar que a expressão entre chaves no lado esquerdo e a expressão do lado direito desta equação possuem sinais contrários. Podemos notar sem dificuldade que a expressão entre as chaves é menor do que zero. De fato, ela corresponde à expressão de $y^{\prime}(A)$ dada pela Eq. 20): como $A>x_{c}$ temos $y^{\prime}(A)<0$ visto que $y(x)$ é decrescente para $x>x_{c}$.

Para mostrar que o lado direito da Eq. 22 é positivo vamos recorrer a seguinte desigualdade logarítmica 8

$$
\ln (1-w) \geqslant-\frac{12 w-12 w^{2}+w^{3}}{6(1-w)(2-w)}
$$


válida para $0 \leqslant w<1$. Pondo $w=\beta A /\left(v_{0} \cos \theta\right)$ nesta desigualdade e multiplicando o resultado por $2 g / \beta^{3}$, temos

$$
\begin{gathered}
\frac{2 g}{\beta^{3}} \ln \left(1-\frac{\beta A}{v_{0} \cos \theta}\right) \geqslant \\
-\frac{A \cdot g}{\beta^{2}}\left[\frac{12 v_{0}^{2} \cos ^{2} \theta-12 \beta A v_{0} \cos \theta+\beta^{2} A^{2}}{3 v_{0} \cos \theta\left(v_{0} \cos \theta-\beta A\right)\left(2 v_{0} \cos \theta-\beta A\right)}\right] .
\end{gathered}
$$

Adicionando a esta desigualdade a expressão

$$
\frac{A \cdot g}{\beta^{2}}\left[\frac{1}{v_{0} \cos \theta}+\frac{1}{v_{0} \cos \theta-\beta A}\right]
$$

obtemos

$$
\begin{array}{r}
\frac{A \cdot g}{\beta^{2}}\left[\frac{1}{v_{0} \cos \theta}+\frac{1}{v_{0} \cos \theta-\beta A}\right]+\frac{2 g}{\beta^{3}} \ln \left(1-\frac{\beta A}{v_{0} \cos \theta}\right) \geqslant \\
\frac{A \cdot g}{\beta^{2}}\left[\frac{3\left(2 v_{0} \cos \theta-\beta A\right)^{2}-\left(12 v_{0}^{2} \cos ^{2} \theta-12 \beta A v_{0} \cos \theta+\beta^{2} A^{2}\right)}{3 v_{0} \cos \theta\left(v_{0} \cos \theta-\beta A\right)\left(2 v_{0} \cos \theta-\beta A\right)}\right]
\end{array}
$$

ou seja,

$$
\begin{array}{r}
\frac{A \cdot g}{\beta^{2}}\left[\frac{1}{v_{0} \cos \theta}+\frac{1}{v_{0} \cos \theta-\beta A}\right]+\frac{2 g}{\beta^{3}} \ln \left(1-\frac{\beta A}{v_{0} \cos \theta}\right) \geqslant \\
\frac{A \cdot g}{\beta^{2}}\left[\frac{2 \beta^{2} A^{2}}{3 v_{0} \cos \theta\left(v_{0} \cos \theta-\beta A\right)\left(2 v_{0} \cos \theta-\beta A\right)}\right]>0,
\end{array}
$$

e, portanto o lado direito da Eq. (22) é positivo.

Enfim, sendo $d A / d \beta<0$ temos que quanto maior $\beta$ (isto é, quanto maior a resistência do ar) menor será o alcance $A$, conforme fisicamente esperado.

\section{Conclusão}

A partir da solução da equação de movimento de uma partícula lançada obliquamente considerando atrito do ar proporcional a velocidade mostramos como esboçar a trajetória da partícula sem recorrer a tabelas ou valores numéricos ou aproximações, não obstante a notável importância de se fazer aproximações em física (e em matemática).

Com este trabalho procuramos apresentar uma proposta didática auxiliar de estudo sobre lançamento oblíquo com resistência do ar ilustrando como conceitos e teoremas matemáticos podem ser usados para deduzir fatos gerais relevantes de um problema físico.

\section{Agradecimentos}

Os autores agradecem ao Departamento de Física da Universidade Regional do Cariri. Agradecem também a Silva Junior "Nanonet" pela confecção das figuras. E também agradecem ao Prof. Dr. Francisco Eduardo de Sousa Filho pelas sugestões.

\section{Referências}

[1] K.R. Symon, Mecânica (Ed. Campus, Rio de Janeiro, 1988), $2^{\mathrm{a}}$ ed.

[2] J.B. Marion and S.T. Thornton, Classical Dynamics of Particles and Systems (Brooks/Cole Pub., New York, 2003), $5^{\text {th }}$ ed.

[3] J. Barcelos Neto, Mecânica Newtoniana, Lagrangiana e Hamiltoniana (Ed. Livraria da Física, 2004).

[4] L. Resende e V. Bonfim, Revista Brasileira de Ensino de Física 30, 3313 (2008).

[5] H. Maceti, C. Levada e I. Lautenschleguer, Cad. Fís. UEFS 10, 7 (2012).

[6] G. Ávila, Cálculo 1 (LTC Ed., Rio de Janeiro, 2003), $7^{\mathrm{a}}$ ed.

[7] E.L. Lima, Análise Real, Volume 1 (IMPA, Rio de Janeiro, 2004).

[8] S. Lang, Short Calculus (Springer-Verlag, New York, 2002), p. 180.

[9] R. Resnick e D. Halliday, Física 1 (LTC Ed., Rio de Janeiro, 1983), $4^{\text {a }}$ ed. 